\title{
Comparison of Stent-Assisted Coiling for Unruptured Internal Carotid Artery Aneurysms Between LVIS or LVIS Jr. and Enterprise VRD: A Retrospective and Single-Center Analysis
}

\author{
Hirotaka SATO'1, Koichi HARAGUCHI² \\ ${ }^{1}$ Asahikawa Medical University, Department of Neurosurgery, Hokkaido, Japan \\ ${ }^{2}$ Hakodate Shintoshi Hospital, Department of Neurosurgery, Hokkaido, Japan \\ Corresponding author: Hirotaka SATO kyokui080021@gmail.com
}

\section{ABSTRACT}

AIM: To compare the low-profile visualized intraluminal support (LVIS or LVIS Jr.) stent, which is a braided microstent, and Enterprise Vascular Reconstructive Device (VRD), which is fabricated using laser cutting technology, in the treatment of internal carotid artery aneurysms.

MATERIAL and METHODS: We investigated 49 unruptured aneurysm cases in which follow-up digital subtraction angiography had been performed. Results of the occlusion were divided into classes 1, 2, and 3 of the Raymond-Roy Occlusion Classification. Statistical significance was defined as $p<0.05$.

RESULTS: In the 49 aneurysm cases, we achieved class 1 in 23 (47\%; LVIS or LVIS Jr., 7; Enterprise, 16; p=0.76), class 2 in 13 (27\%; LVIS or LVIS Jr., 5; Enterprise, 8; p=0.74), and class 3 in 13 (27\%; LVIS or LVIS Jr., 5; Enterprise, 8; p=0.74). Based on the follow-up imaging of the 49 aneurysms, we achieved class 1 in 32 cases (65\%; LVIS or LVIS Jr., 16; Enterprise, 16; $p<0.01)$, class 2 in 7 (14\%; LVIS or LVIS Jr., 0; Enterprise, 7; $p<0.01$ ), and class 3 in 10 (20\%; LVIS or LVIS Jr., 1; Enterprise, 9; $p=0.13$ ).

CONCLUSION: Recently, the flow diversion effect of stents has garnered more attention compared to coil embolization. LVIS or LVIS Jr. exerts a higher flow diversion effect than other stents and may improve the Raymond-Roy Occlusion Classification. In the future, the role of stents in the treatment of aneurysms will become more important.

KEYWORDS: Stent-assisted coiling, Low-profile visualized intraluminal support, Enterprise VRD, Internal carotid artery aneurysms

\section{INTRODUCTION}

E Indovascular coil embolization has been widely performed to treat intracranial aneurysms. However, Janeurysms with wide necks or complex morphologies are difficult to treat with simple endovascular coil embolization. For such aneurysms, stent-assisted coiling (SAC) is performed. According to a previous report, SAC results in outcomes similar to those obtained with simple endovascular coil embolization, despite aneurysms treated with SAC having more difficult morphologies than those treated with simple endovascular coil embolization (4). Stents have the theoretical advantage of providing an anatomical barrier and remodeling the blood flow for endothelial growth and vessel wall healing $(1,3,15)$.

Although several studies have reported on aneurysms treated with flow diverter stents (9), SAC is considered a useful technique for treating aneurysms with wide necks or complex morphologies. Stents can be broadly divided into two groups. The first group comprises the Neuroform Atlas Stent (Stryker, Fremont, California, USA) and Enterprise Vascular Reconstruction Device (VRD) stents (Codman \& Shurtleff, Inc., Raynham, Massachusetts, USA) that are nitinol stents manufactured using laser cutting. They can be inserted through standard microcatheters and used for 
SAC. In particular, the Enterprise VRD stents are closed-cell stents. The second group comprises low-profile visualized intraluminal support stents (LVIS/LVIS Jr.; MicroVention, Inc., Terumo, Aliso Viejo, CA, USA), which are self-expanding braided microstents and closed-cell stents (8). Although these two groups of stents have been compared in previous reports (17), only a few studies have compared them based on the location of the aneurysm.

Therefore, the present study aimed to investigate the outcomes of stent-assisted endovascular coil embolization in treating unruptured internal carotid artery (ICA) aneurysms, and to compare and assess embolism progression and complications in the short term following the use of the LVIS/ LVIS Jr. and Enterprise VRD stents.

\section{MATERIAL and METHODS}

This retrospective study was conducted between October 2010 and March 2019, and included 70 unruptured ICA aneurysms treated with SAC. We investigated 49 unruptured aneurysm cases in which follow-up digital subtraction angiography (DSA) was performed, approximately 6-12 months after SAC. The surgical indications reported in the Japanese Guideline for the Management of Stroke 2015 for unruptured intracranial aneurysms were followed. These guidelines recommend that aneurysms of 5-7 $\mathrm{mm}$ be treated. In addition, these guidelines recommend that even if the aneurysms are $<5 \mathrm{~mm}$ but are located in the posterior circulation or anterior communication artery or ICA-posterior communicating artery (Pcom) or have high dome-neck ratios, with irregular shapes or blebs, they should be treated. In the present study, the dome-neck ratio of all the aneurysms was $\leq 2$.

The recorded variables were patient age and sex, stent type, maximum aneurysm size, dome-neck ratio, location of the aneurysm, findings of the initial occlusion, procedure-related postoperative infarctions, and results of the follow-up DSA.

\section{Endovascular Procedure}

All procedures were performed under general anesthesia. Dual antiplatelet therapy was administered to all the patients 7 days before the procedure. Antiplatelet medications were continued for 3 postoperative months in all the patients.

We used Enterprise VRD stents between October 2010 and November 2015 and LVIS/LVIS Jr. stents between May 2016 and March 2019 and inserted them using an 8-Fr balloon guide catheter in all patients. We have been using LVIS as the first choice since May 2016 for ICA aneurysms, expecting the flow diverter effect of LVIS. Headway 17 STR microcatheters (MicroVention, Inc., Terumo, Aliso Viejo, CA, USA) and Headway 21 STR microcatheters (MicroVention, Inc., Terumo, Aliso Viejo, CA, USA) were used for LVIS/LVIS Jr. stents for coiling, whereas Excelsior SL-10 STR microcatheters (Stryker, Fremont, California, USA), Echelon-10 STR microcatheters (Medtronic, Irvine, CA, U.S.A.), and Prowler Select Plus microcatheters (Codman Neurovascular, Johnson \& Johnson, Miami, Florida, USA) were used for Enterprise VRD stents for coiling. In all the patients, the aneurysms were embolized to classify them into class 3 aneurysms.

\section{Outcome}

Outcomes of the occlusion were divided into classes 1, 2, and 3 of the Raymond-Roy occlusion classification (13). We collected these outcome data from the operative notes of the neurosurgeon. Follow-up data were also divided into classes 1,2 , and 3 of the Raymond-Roy occlusion classification by the neurosurgeon. This neurosurgeon was a doctor who had not participated in the operation, but was aware of the type of stent before deciding the classification of the outcomes/data. DSA was performed after approximately 6-12 postoperative months.

\section{Statistical Analysis}

Variables are expressed as mean \pm standard deviation, median [interquartile range (IQR)], and the number of patients (\%), as appropriate. The Pearson's $X^{2}$ test or Fisher's exact test was used to assess the associations between categorical variables. For continuous variables, the Kolmogorov-Smirnov test was used to determine if the distributions were normal. For normal distributions, the data were analyzed using the $t$-test or Mann-Whitney U-test, as appropriate. $P$-values of $<0.05$ were considered statistically significant.

All statistical analyses were conducted using EZR (Saitama Medical Center, Jichi Medical University, Saitama, Japan), which is a graphical user interface for $R$ (version 3.5.1) (6).

\section{RESULTS}

Table I summarizes the clinical and demographic data of the patient population. The patient population comprised 42 women and 7 men, with a mean age of $58.9 \pm 13.4$ years. The median aneurysm size was $6.5 \mathrm{~mm}$ (IQR, 5.1-7.5 mm), and the dome-neck ratio was 1.1 (IQR, 1.0-1.4). LVIS/LVIS Jr. stents were used in 17 patients (35\%), whereas Enterprise VRD stents were used in 32 (65\%). Overall, 18 (37\%), 16 (33\%), and 15 aneurysms were located in the superior hypophyseal artery (SHA)-ICA, ophthalmic artery (OA)-ICA, and Pcom-ICA, respectively. There was no difference between the LVIS/LVIS Jr. and Enterprise VRD stent groups in terms of the mean patient age $(p=0.80)$, patient sex $(p=0.40)$, or maximum size of the aneurysm $(p=0.11)$. However, the dome-neck ratio $(p=0.01)$ was significantly different between the LVIS/LVIS Jr. and Enterprise VRD stent groups.

\section{Procedural Results}

Of the 49 aneurysms, 23 (47\%) were classified as class 1 aneurysms ( 7 for the LVIS/LVIS Jr. stents and 16 for Enterprise VRD stents; $p=0.76), 13$ (27\%) as class 2 (5 for LVIS/LVIS Jr. stents and 8 for Enterprise VRD stents; $p=0.74)$, and $13(27 \%)$ as class 3 (5 for LVIS/LVIS Jr. stents and 8 for Enterprise VRD stents; $p=0.74$ ) (Table II). Symptomatic infarction was $10 \%$ $(n=5)$ ( 3 for the LVIS/LVIS Jr. stents and 2 for the Enterprise VRD stents; $p=0.32)$. Overall, two patients treated with SAC using the LVIS/LVIS Jr. stent had transient paralysis, and one had right upper limb paralysis. Conversely, one patient treated with SAC using the Enterprise VRD stent had right upper limb paralysis, and one had unilateral spatial neglect. The total permanent morbidity rate related to SAC was $6 \%(n=3)(1$ for the LVIS/LVIS Jr. stent and 2 for Enterprise VRD stents; $p=1$ ). 


\section{Radiological Follow-Up Results}

Based on the results of the follow-up DSA after approximately 6-12 postoperative months, 32 (65\%) aneurysms were classified as class 1 ( 16 for LVIS/LVIS Jr. stents and 16 for Enterprise VRD stents; $p<0.01), 7(14 \%)$ as class 2 ( 0 for the LVIS/LVIS Jr. stent and 7 for Enterprise VRD stents; $p<0.01$ ), and $10(20 \%)$ as class 3 ( 1 for the LVIS/LVIS Jr. stent and 9 for the Enterprise VRD stents; $p=0.13$ ) (Table III).

Table I: Characteristics of Patients with Aneurysms Undergoing Stent and Coil Embolization

\begin{tabular}{|c|c|}
\hline Characteristic & Value \\
\hline Mean age (years) (mean \pm SD) & $58.88 \pm 13.4$ \\
\hline Gender (female:male) & $42: 7$ \\
\hline \multicolumn{2}{|l|}{ Aneurysm location } \\
\hline ICA-SHA & $18(37 \%)$ \\
\hline ICA-Opth & $16(33 \%)$ \\
\hline ICA-Pcom & $15(31 \%)$ \\
\hline Aneurysm size & \multirow{2}{*}{$6.5(5.1-7.5)$} \\
\hline Median (IQR) (mm) & \\
\hline Dome to neck ratio & \multirow{2}{*}{$1.1(1.0-1.4)$} \\
\hline Median (IQR) (mm) & \\
\hline \multicolumn{2}{|l|}{ Stent type } \\
\hline LVIS or LVIS Jr. & 17 (35\%) \\
\hline Enterprise & $32(65 \%)$ \\
\hline Morbidity & $3(6 \%)$ \\
\hline Mortality & 0 \\
\hline
\end{tabular}

\section{DISCUSSION}

Recently, the flow diversion effect of stents has garnered increasing attention compared to coil embolization. SAC is useful for treating aneurysms because it can reduce the recurrence rate and offer benefits with respect to the mechanical barrier, flow diversion effect, and biological effects (5).

In the present study, we compared LVIS/LVIS Jr. and Enterprise VRD stents placed in aneurysms located in the ICA. Both stents have characteristics of a closed-cell type. However, each stent is prepared by a different method. Therefore, we compared LVIS/LVIS Jr. and Enterprise VRD stents placed in aneurysms located in the ICA with many sidewall aneurysms, except ICA top aneurysms.

\section{Study Analysis}

The LVIS/LVIS Jr. stent is excellent in managing aneurysms, as reported in several studies. Feng et al. reported that the occurrence rates of complete and near-complete occlusions were similar with both LVIS/LVIS Jr. and Enterprise VRD stents (2). Moreover, the LVIS/LVIS Jr. stent was associated with lower rates of recanalization and in-stent stenosis, although the difference between LVIS/LVIS Jr. and Enterprise VRD stents was not significant. $\mathrm{Li}$ et al. conducted a computed fluid dynamics study and compared the treatment of mediumsized intracranial aneurysms with LVIS/LVIS Jr. and Enterprise VRD embolization to determine the effects of hemodynamic changes caused by different stents and coil packing densities (7); they found that SAC embolization using LVIS/LVIS Jr. stents showed a lower rate of recanalization after endovascular treatment of medium-sized intracranial aneurysms.

The findings of the present study are consistent with these previously reported findings. It is noteworthy that we found no significant difference in the number of class 1 aneurysms immediately after surgery but found a significant difference in the follow-up DSA. Class 2 aneurysms can be found improved

Table II: Comparison of the Characteristics of LVIS or LVIS Jr. and Enterprise About Patients and Morphology, Procedual Results

\begin{tabular}{lccc}
\hline & LVIS or LVIS Jr. (n=17) & Enterprise $(\mathbf{n}=\mathbf{3 2})$ & $\mathbf{p}$ \\
\hline Mean age $($ mean \pm SD) (years) & $58.18 \pm 9.25$ & $59.25 \pm 15.46$ & 0.80 \\
\hline Gender (female) & 16 & 26 & 0.40 \\
\hline Aneursysm size median (IQR) (mm) & $5.2(5.0-6.9)$ & $6.6(5.3-7.5)$ & 0.11 \\
\hline Dome to neck ratio* median (IQR) $(\mathrm{mm})$ & $1.09(0.92-1.3)$ & $1.21(1.13-1.66)$ & $\mathbf{0 . 0 1}$ \\
\hline Initial results & & & \\
\hline \multicolumn{1}{l}{ Class 1 } & 7 & 16 & 0.76 \\
\hline \multicolumn{1}{c}{ Class 2 } & 5 & 8 & 0.74 \\
\hline \multicolumn{1}{c}{ Class 3 } & 5 & 8 & 0.74 \\
\hline Symptomatic infarction (including transistent symptom) & 3 & 2 & 0.32 \\
\hline Morbidity & 1 & 2 & 1 \\
\hline
\end{tabular}

*Variables showing significant diferences. SD: Standard deviation, IQR: Interquartile range. 
to class 1 aneurysms on follow-up DSA (Figure 1A-F). This result may be attributed to the fact that the LVIS/LVIS Jr. stent exerts a flow diversion effect, although the LVIS/LVIS Jr. group has a lower dome-neck ratio. In other words, it may be less

Table III: Comparison of the Characteristics of LVIS or LVIS Jr. and Enterprise About Radiological Follow-up Results

\begin{tabular}{cccc}
\hline & $\begin{array}{c}\text { LVIS or LVIS Jr. Enterprise } \\
(\mathbf{n}=\mathbf{1 7})\end{array}$ & $\mathbf{p}$ \\
\hline Follow-up results & & & \\
\hline Class 1 $^{*}$ & 16 & 16 & $<0.01$ \\
\hline Class 2 & 0 & 7 & 0.07 \\
\hline Class 3 & 1 & 9 & 0.13 \\
\hline
\end{tabular}

*Variables showing significant diferences. necessary to embolize the coil to the maximum extent during the operation, thus reducing the risk of intraoperative rupture. We encountered a case of aneurysm wherein the "eclipse sign" is recognized after placing the LVIS/LVIS Jr. stent (Figure $2 \mathrm{~A}-\mathrm{C})$. This result elucidated the advantage of LVIS/LVIS Jr., and we think that the efficacy of LVIS/LVIS Jr. will become apparent after 6-12 months.

\section{Clinical Impact}

In recent years, Neuroform stents have been improved to Neuroform Atlas stents. This stent has very good adhesion to blood vessels but has a metal coverage rate of $10 \%-11 \%$ $(11,16)$, which is inferior to the $15 \%-22 \%$ metal coverage rate of LVIS Jr. and $18 \%-29 \%$ of LVIS. LVIS/LVIS Jr. stents have excellent characteristics owing to their high metal coverage rate $(11,16)$. In other words, Neuroform Atlas, which is an opencell stent, is considered to be advantageous in aneurysms wherein blood vessels are strongly bent and adhesion is

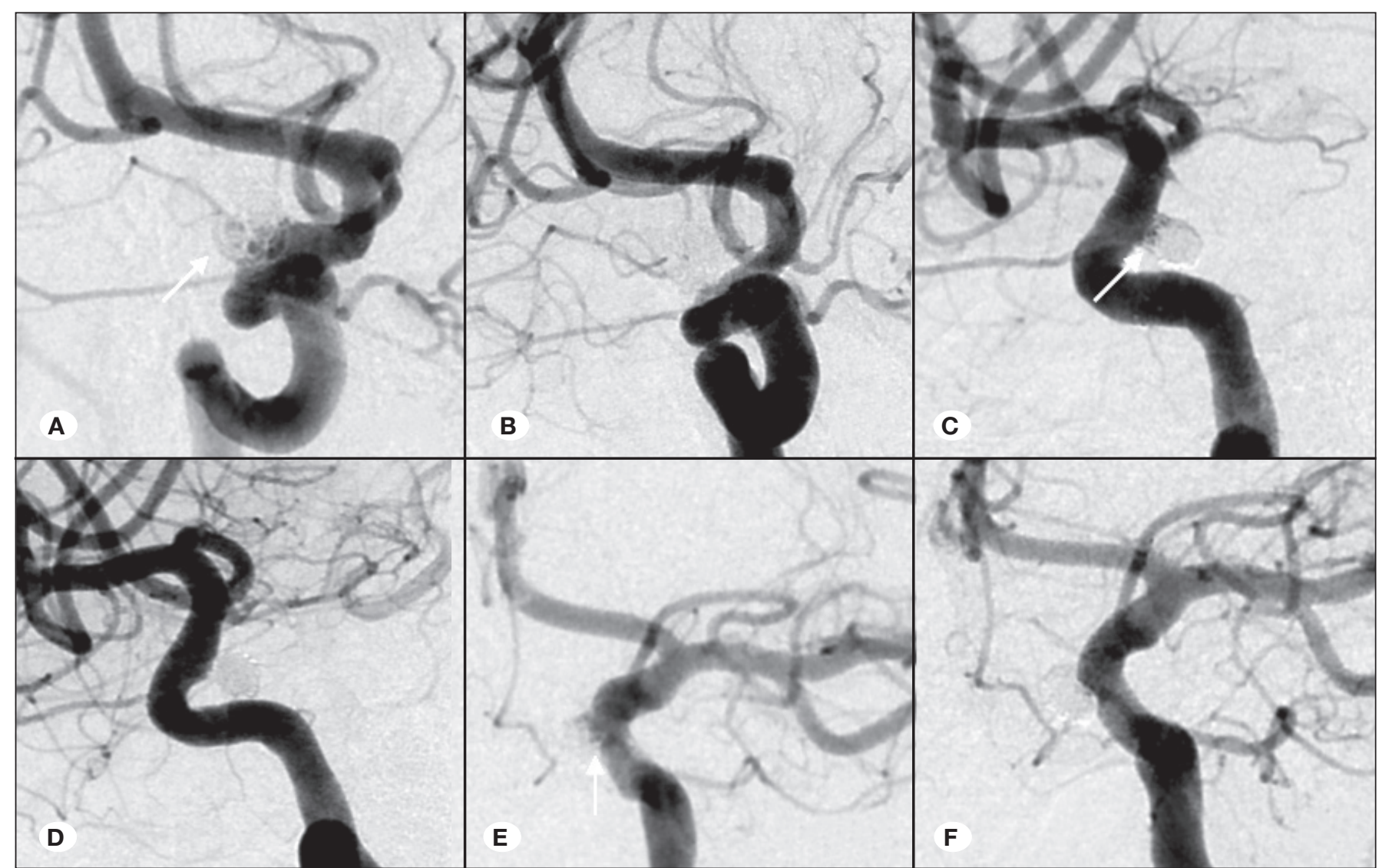

Figure 1: Cases in which improvement was observed on follow-up digital subtraction angiography (DSA). (A) and (B) A case of an aneurysm in the ophthalmic artery (OA)-internal carotid artery (ICA). Although stent-assisted coiling (SAC) was performed, the inflow of the contrast medium into the dome was observed immediately after surgery (A). We used the low-profile visualized intraluminal support (LVIS) stent $(4.5 \times 23 \mathrm{~mm})$. However, this case was improved to Raymond-Roy class 1 at the time of follow-up DSA (B).

(C) and (D) A case of an aneurysm in the superior hypophyseal artery (SHA)-ICA. Although SAC was performed, the neck remnant was observed immediately after surgery $(C)$. We used the LVIS stent $(4.5 \times 18 \mathrm{~mm})$. However, this case was improved to Raymond-Roy class 1 at the time of follow-up DSA (D).

(E) and (F) A case of an aneurysm in the SHA-ICA. Although SAC was performed, the neck remnant was observed immediately after surgery (E). We used the LVIS stent $(3.5 \times 22 \mathrm{~mm})$. However, this case was improved to Raymond-Roy class 1 at the time of follow-up DSA (F). 


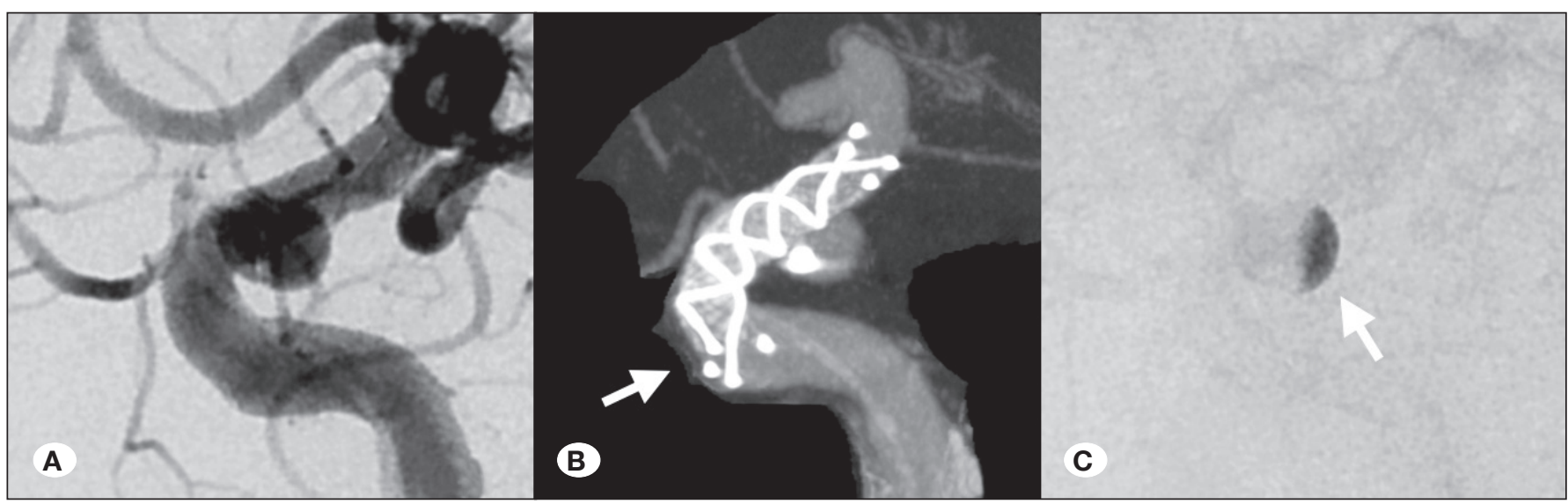

Figure 2: A) A case of the posterior communicating artery, which is a branch of the internal carotid artery. B) We placed the low-profile visualized intraluminal support (LVIS) stent $(3.5 \times 17 \mathrm{~mm})$ before coil embolization. The arrow indicates the LVIS stent. C) We could confirm the "eclipse sign" simply by detection.

difficult. However, we think that LVIS stents should be used in cases in which these stents can be used without any difficulty. SAC using LVIS stents in small aneurysms with morphological characteristics of a wide neck is likely to provide excellent outcomes.

However, this feature can be considered a risk factor for thrombosis. SAC increases the possibility of thrombosis (10). In the present study, there was no significant difference in thrombosis between the LVIS/LVIS Jr. and Enterprise VRD stent groups. In the LVIS group, three patients (17\%) experiences a stroke, including transient symptoms. Two patients had transient paralysis but were discharged with a modified Rankin scale score of 0 . Morbidity was observed in one patient (5.9\%). In the Enterprise VRD group, both stroke and morbidity were observed in 2 patients $(6.2 \%)$. The incidence rate of stroke in patients treated with LVIS was slightly higher than in those treated with Enterprise VRD. However, there were two transient cases, and the morbidity rate was low at $5.9 \%$. The incidence rate of stroke increased slightly due to the small number of patients being treated with LVIS. Considering these findings, we think that the results of our study are acceptable compared with those reported by previous studies (14). Taken together, this meta-analysis reported that thromboembolic issues were $10 \%$ (14). However, we did not perform platelet function tests during this time because these tests were not covered by insurance; nevertheless, we are testing platelet function at present. Therefore, we think that thrombotic complications in patients using LVIS can be reduced. In an effort to reduce the risk of thrombosis associated with LVIS/LVIS Jr. stents, we must be careful about using antiplatelet drugs.

\section{Limitations}

The present study has some limitations. First, the initial coil packing density of the aneurysm may be responsible for the degree of recanalization (12). However, we did not compare the LVIS/LVIS Jr. and Enterprise VRD stents in this regard. Second, the study was not blinded because the neurosurgeon who evaluated the results was aware of the type of stent, indicating that this result may be mixed intentionally. Third, although all patients received two antiplatelet drugs, platelet function tests were not included in our clinical practice during the study period, indicating that the accuracy of the data on thrombosis is uncertain.

\section{CONCLUSION}

The postoperative outcomes of LVIS/LVIS Jr. and Enterprise VRD stents for treating aneurysms located in the ICA were not different, except when treating ICA top aneurysms; however, the postoperative outcomes of LVIS/LVIS Jr. stents improved after 6-12 months postoperatively.

\section{REFERENCES}

1. Fargen KM, Hoh BL, Welch BG, Pride GL, Lanzino G, Boulos AS, Carpenter JS, Rai A, Veznedaroglu E, Ringer A, Rodriguez-Mercado R, Kan P, Siddiqui A, Levy El, Mocco $\mathrm{J}$ : Long-term results of enterprise stent-assisted coiling of cerebral aneurysms. Neurosurgery 71:239-244; discussion 244, 2012

2. Feng X, Qian Z, Liu P, Zhang B, Wang L, Guo E, Wen X, Xu W, Jiang C, Wu Z, Li Y, Liu A: Comparison of recanalization and instent stenosis between the low-profile visualized intraluminal support stent and enterprise stent-assisted coiling for 254 intracranial aneurysms. World Neurosurg 109:e99-e104, 2018

3. Geyik S, Yavuz K, Yurttutan N, Saatci I, Cekirge HS: Stentassisted coiling in endovascular treatment of 500 consecutive cerebral aneurysms with long-term follow-up. AJNR Am J Neuroradiol 34:2157-2162, 2013

4. Hetts SW, TurkA, English JD, Dowd CF, Mocco J, Prestigiacomo C, Nesbit G, Ge SG, Jin JN, Carroll K, Murayama Y, Gholkar A, Barnwell S, Lopes D, Johnston SC, McDougall C, Matrix, Platinum Science Trial I: Stent-assisted coiling versus coiling alone in unruptured intracranial aneurysms in the matrix and platinum science trial: Safety, efficacy, and mid-term outcomes. AJNR Am J Neuroradiol 35:698-705, 2014

5. Hong Y, Wang YJ, Deng Z, Wu Q, Zhang JM: Stent-assisted coiling versus coiling in treatment of intracranial aneurysm: A systematic review and meta-analysis. PLoS One 9:e82311, 2014 
6. Kanda $Y$ : Investigation of the freely available easy-to-use software 'EZR' for medical statistics. Bone Marrow Transplant 48:452-458, 2013

7. Li W, Wang Y, Zhang Y, Wang K, Zhang Y, Tian Z, Yang X, Liu J: Efficacy of LVIS vs. Enterprise stent for endovascular treatment of medium-sized intracranial aneurysms: A hemodynamic comparison study. Front Neurol 10:522, 2019

8. Mohlenbruch M, Herweh $\mathrm{C}$, Behrens L, Jestaedt L, Amiri $\mathrm{H}$, Ringleb PA, Bendszus M, Pham M: The LVIS Jr. microstent to assist coil embolization of wide-neck intracranial aneurysms: Clinical study to assess safety and efficacy. Neuroradiology 56:389-395, 2014

9. Nelson PK, Lylyk P, Szikora I, Wetzel SG, Wanke I, Fiorella D: The pipeline embolization device for the intracranial treatment of aneurysms trial. AJNR Am J Neuroradiol 32:34-40, 2011

10. Phan K, Huo YR, Jia F, Phan S, Rao PJ, Mobbs RJ, Mortimer AM: Meta-analysis of stent-assisted coiling versus coiling-only for the treatment of intracranial aneurysms. J Clin Neurosci 31:15-22, 2016

11. Poncyljusz W, Bilinski P, Safranow K, Baron J, Zbroszczyk M, Jaworski M, Bereza S, Burke TH: The LVIS/LVIS Jr. stents in the treatment of wide-neck intracranial aneurysms: multicentre registry. J Neurointerv Surg 7:524-529, 2015
12. Raymond J, Guilbert F, Weill A, Georganos SA, Juravsky L, Lambert A, Lamoureux J, Chagnon M, Roy D: Long-term angiographic recurrences after selective endovascular treatment of aneurysms with detachable coils. Stroke 34:1398-1403, 2003

13. Roy D, Milot G, Raymond J: Endovascular treatment of unruptured aneurysms. Stroke 32:1998-2004, 2001

14. Shapiro M, Becske T, Sahlein D, Babb J, Nelson PK: Stentsupported aneurysm coiling: A literature survey of treatment and follow-up. AJNR Am J Neuroradiol 33:159-163, 2012

15. Spiotta AM, Wheeler AM, Smithason S, Hui F, Moskowitz S: Comparison of techniques for stent assisted coil embolization of aneurysms. J Neurointerv Surg 4:339-344, 2012

16. Wang C, Tian Z, Liu J, Jing L, Paliwal N, Wang S, Zhang $Y$, Xiang J, Siddiqui AH, Meng $H$, Yang X: Flow diverter effect of LVIS stent on cerebral aneurysm hemodynamics: A comparison with Enterprise stents and the Pipeline device. J Transl Med 14:199, 2016

17. Wang J, Vargas J, Spiotta A, Chaudry I, Turner RD, Lena J, Turk A: Stent-assisted coiling of cerebral aneurysms: A singlecenter clinical and angiographic analysis. J Neurointerv Surg 10:687-692, 2018 